\title{
ORIENTAÇÕES ESTRATÉGICAS E DESEMPENHO: EVIDÊNCIAS DO SETOR VAREJISTA DE ÓPTICAS
}

\section{Giana de Vargas Mores}

Mestre em Agronegócios na Universidade Federal do Rio Grande do Sul - UFRGS

Professora da Universidade Comunitária da Região de Chapecó - UNOCHAPECÓ gimores@gmail.com (Brasil)

\section{Eduardo Botti Abbade}

Doutorando em Agronegócios na Universidade Federal do Rio Grande do Sul - UFRGS

Professor do Centro Universitário Franciscano - UNIFRA e da Faculdade Palotina de Santa Maria FAPAS

eduardo@unifra.br (Brasil)

\section{Verner Luis Antoni}

Doutor em Engenharia de Produção pela Universidade Federal de Santa Catarina - UFSC

Professor da Universidade de Passo Fundo - UPF

antoni@upf.br(Brasil)

\section{RESUMO}

As orientações para o mercado e para a aprendizagem interferem no desempenho organizacional por meio dos impactos diretos na inovação (Baker \& Sinkula, 1999). O objetivo deste estudo consistiu em avaliar as orientações para o mercado e aprendizagem e a inovação impactando no desempenho das ópticas de Passo Fundo/RS. Este estudo é caracterizado como um survey com adesão de 24 ópticas, em que os dados foram analisados por meio de análises descritivas e multivariadas. Na análise multivariada e na modelagem de equações estruturais, o método de estimação utilizado foi o Partial Least Squares. Os resultados sugerem que a orientação para mercado e a inovação impactam de forma positiva no desempenho. Observou-se também que, de um lado, a orientação para aprendizagem impacta positivamente na orientação para o mercado e, por outro, apresenta impacto negativo na inovação. Considera-se que estudos adicionais com setores empresariais específicos são necessários.

Palavras-chave: Desempenho organizacional; inovação; orientação para aprendizagem; orientação para mercado. 


\section{INTRODUÇÃO}

A orientação para o mercado passou a receber maior atenção dos pesquisadores na literatura nos anos de 1990, principalmente em pesquisas realizadas com organizações de grande porte dos Estados Unidos e da Europa (Kohli \& Jaworski, 1990; Day, 1994; Baker \& Sinkula, 1999). Na década de 1990, os autores focavam seus estudos para o contexto de que a rentabilidade era resultado da orientação para o mercado, contando com uma cultura voltada para o cliente (Kohli \& Jaworski, 1990), a fim de manter uma vantagem competitiva sustentável no longo prazo. Kohli e Jaworski (1990) descrevem que a orientação para o mercado é resultado da combinação de geração de inteligência de mercado, disseminação da inteligência e capacidade de resposta ao mercado por parte da organização, porém, ligada a uma cultura organizacional de aprendizado contínuo (Antoni, 2004). Na organização, Sinkula, Baker e Noordewier (1997) evidenciam que a orientação para aprendizagem é resultado do compromisso com aprendizagem, postura de mente aberta e visão compartilhada.

Para manter-se no mercado e aumentar a satisfação de seus clientes, uma organização necessita estar orientada para o mercado, a fim de identificar as necessidades e expectativas de seus clientes, bem como de formular e definir suas estratégias, adaptando-se rapidamente às oscilações do mercado e às preferências do consumidor. Perin, Sampaio e Faleiro (2004) destacam que a inovação se torna fundamental para um desempenho superior organizacional no longo prazo. As orientações para o mercado e para aprendizagem interferem no desempenho da organização pelos efeitos diretos causados sobre a inovação (Baker \& Sinkula, 1999).

De acordo com Antoni (2004), em nível nacional, há poucos estudos que evidenciam a orientação para o mercado e sua relação com o desempenho organizacional no contexto de serviços. Dessa forma, surge a necessidade de um olhar aprofundado para a avaliação do grau de orientação para o mercado, orientação para aprendizagem e inovação do setor de serviços brasileiro, dada sua significativa importância para a composição do produto interno bruto do país (desempenho econômico).

Nesse contexto, este trabalho busca analisar o segmento óptico varejista da cidade de Passo Fundo/RS e conta, para tal, com o apoio da Câmara de Dirigentes Lojistas (CDL). O segmento de ópticas passo-fundenses foi escolhido a critério dos pesquisadores pelo fato de apresentar um processo dinâmico de mudanças com alto índice de competitividade e por trabalhar com novas tecnologias de produtos.

Com base nas afirmações apresentadas, o objetivo geral desta pesquisa consiste em descrever as relações existentes entre a orientação para mercado, orientação para aprendizagem, inovação e

Revista de Administração e Inovação, São Paulo, v. 10, n.3, p.65-88, jul./set. 2013. 
desempenho das organizações do segmento analisado. $\mathrm{O}$ estudo objetiva também testar os modelos de referência propostos, verificando a influência das orientações para o mercado e para a aprendizagem na inovação e no desempenho das organizações estudadas. As contribuições passam pela linha do experimento em ambientes diferenciados, em organizações de menor porte, tendo o setor varejista como ênfase.

Por focar sua amostra em um setor varejista específico, esta pesquisa apresenta contribuições científicas restritas, sendo, dessa forma, direcionada ao público empresarial dos setores óptico e correlatos. As evidências apresentadas podem sugerir melhores condições na tomada de decisão e na articulação das orientações estratégicas abordadas na pesquisa, que também se direciona ao público acadêmico-científico, uma vez que as evidências obtidas no setor varejista de ópticas contribuem para a construção do conhecimento científico, buscando consolidar as já existentes acerca dos impactos das orientações para o mercado e aprendizagem e da inovação no desempenho organizacional.

\section{EMBASAMENTO TEÓRICO-EMPÍRICO}

Esta seção está organizada em quatro subseções: a primeira aborda a orientação para o mercado; a segunda faz referência à orientação para a aprendizagem; a terceira se dedica à análise da inovação; e, por fim, na quarta subseção, são relacionadas as três temáticas referidas.

\subsection{Orientação para o mercado}

A orientação para o mercado, quando utilizada em uma organização, "permite uma performance superior nos negócios" (Antoni, 2004, p. 06). Day (1994a) sinaliza que as organizações devem se antecipar às mudanças que ocorrem no mercado e responder a elas antes de seus concorrentes. $\mathrm{O}$ mesmo autor frisa que a orientação para o mercado auxilia no entendimento do que gera satisfação para o cliente, o que permite à organização ter desempenho superior ao de seus concorrentes.

Em seu estudo, Kohli e Jaworski (1990) realizaram uma pesquisa por meio de entrevistas com gerentes, em que os entrevistados avaliaram a base da visão de marketing. Na visão dos entrevistados, os resultados obtidos foram: foco no consumidor é o elemento central da orientação para o mercado e as ações devem ser tomadas com base na percepção do consumidor, bem como na inteligência de marketing; a orientação para o mercado não está somente sob a responsabilidade do departamento de marketing; a

Revista de Administração e Inovação, São Paulo, v. 10, n.3, p.65-88, jul./set. 2013. 
rentabilidade aparece como consequência da orientação para o mercado. Segundo esses autores, o resultado da orientação para o mercado é o melhor desempenho da organização nos negócios e, como consequência, a rentabilidade.

Narver e Slater (1990) destacam que a orientação para o mercado gera vantagens competitivas sustentáveis ao considerar o comportamento do consumidor para definição de estratégias. Adicionam a essa análise o contexto da concorrência, ou seja, desempenho superior ao dos concorrentes. Dessa forma, a vantagem competitiva sustentável surge ao se criar valor superior para os clientes-alvo, identificando suas necessidades e expectativas atuais e futuras, considerando análises de longo prazo e de rentabilidade.

Kohli e Jaworski (1993) apontam que a orientação para o mercado está relacionada à ênfase na orientação dada pela alta administração, aversão ao risco dos gestores, conflitos entre os departamentos da organização e à centralização. A escala Markor (Market orientation) para mensuração da orientação para o mercado nas organizações foi elaborada por Kohli, Jaworski e Kumar (1993). Essa escala de avaliação é dividida em três dimensões: i) geração de inteligência de mercado; ii) disseminação da inteligência; iii) capacidade de resposta da organização ao mercado ou responsividade.

A geração de inteligência compreende pesquisas com consumidores e mecanismos complementares, tais como reuniões com clientes, discussões com parceiros e análise de relatórios de vendas. A tentativa de envolver a análise dos fatores externos na geração de inteligência está ligada à necessidade de conhecer as ações dos competidores e a maneira como elas influenciam as preferências dos clientes. Outro fator que influencia na geração de inteligência é o ambiente dos negócios (Kohli, Jaworski e Kumar, 1993).

A disseminação de inteligência deve ser realizada por meio de comunicação horizontal, a qual deve ocorrer dentro da organização, envolvendo todos os departamentos (Kohli \& Jaworski, 1990). Considera-se também que os fluxos de informações verticais e diagonais desempenham papel fundamental para essa disseminação, associados à comunicação horizontal. A responsividade relaciona-se com as ações adotadas para responder à geração e à disseminação de inteligência. Envolve a seleção dos mercados-alvo, o desenho e a oferta de produtos que respondam às necessidades atuais e futuras dos clientes, etc. (Kohli et al., 1993).

Pela visão de Day (1994b), compreende-se que a orientação para o mercado está relacionada com aprendizagem organizacional, considerando um processo contínuo de monitoramento. Para Antoni (2004, p. 59), faz-se necessário "um ambiente organizacional que promova o comprometimento de todos os níveis organizacionais, para que, com isso, ocorram a implementação e a manutenção de uma 'cultura' de orientação para o mercado".

Revista de Administração e Inovação, São Paulo, v. 10, n.3, p.65-88, jul./set. 2013. 


\subsection{Orientação para aprendizagem}

Perin et al. (2004, p. 82) definem orientação para aprendizagem como "um conjunto de valores organizacionais que influenciam a propensão da organização para criar e utilizar o conhecimento". Nesse sentido, Sinkula et al. (1997, p. 83) abordam três valores da organização que estão "associados à predisposição da organização em aprender: compromisso com a aprendizagem, visão compartilhada e postura de mente aberta".

O comprometimento com a aprendizagem faz referência à disposição dos indivíduos em aprender em conjunto num ambiente propício para o desenvolvimento da aprendizagem organizacional. Dessa forma, o grau de comprometimento com a aprendizagem organizacional é considerado um importante ativo das organizações. A visão compartilhada remete à concentração e dedicação de todos os membros da organização na aprendizagem, o que resulta em um fortalecimento da sua força, bem como em comprometimento e intencionalidade. A mente aberta está relacionada à avaliação crítica das operações e atividades da organização por parte de seus membros, assim como à aceitação de novas ideias e propostas de melhoria (Sinkula, Baker e Noordewier, 1997).

“As organizações estão enfrentando uma competição crescente e se deparam com o desafio de criar uma combinação de cultura e clima organizacional que as orientem para o mercado com um processo de aprendizagem constante", tendo como objetivo principal a criação de "valor superior para os clientes, obtendo vantagens competitivas sustentáveis" (Antoni, 2004, p. 04).

Sinkula et al. (1997) abordam que a aprendizagem organizacional é vista como um processo cíclico. Na visão desses autores, são considerados como facilitadores para maximizar a aprendizagem da organização: os valores organizacionais, o processamento das informações do comportamento de mercado e a tomada de ação por parte da organização. Ainda, concluem que níveis altos de aprendizagem resultam no aumento da geração e disseminação da informação de mercado.

A geração de informações de mercado é forte determinante no processo de aprendizagem organizacional e pode ter caráter interno ou pode ocorrer externamente à organização, segundo Perin, Sampaio, Baker e Simões (2009). No mesmo estudo, evidencia-se que a aprendizagem corrobora para o desenvolvimento de novos produtos.

Revista de Administração e Inovação, São Paulo, v. 10, n.3, p.65-88, jul./set. 2013. 


\subsection{Inovação}

Na literatura, conforme abordado por Perin et al. (2004), considera-se a inovação como elemento principal para haver vantagem competitiva sustentável e desempenho organizacional superior no longo prazo. Os mesmos autores citam como facilitadores da inovação: "a coordenação e relação interfuncional, a capacidade de incentivar e recompensar ideias, a atitude gerencial positiva em relação à mudança e a cultura mais voltada para o mercado" (2004, p. 84). "Inovação é definida como a geração, aceitação e implementação de novas ideias, processos, produtos ou serviços" (Erdil, Erdil e Keskin, 2003, p. 4, tradução nossa).

Nessa perspectiva, Erdil, Erdil e Keskin (2003) sustentam que a orientação para o mercado influencia no grau de inovação e no desempenho da organização. Assim, a inovação oportuniza o crescimento e a expansão da organização para obter vantagem competitiva, segundo os autores. Hanvanich, Sivakumar e Hult (2006) argumentam que as relações entre orientação para aprendizagem e memória da organização, e inovação e performance da organização alteram o grau de turbulência do ambiente no qual as organizações estão expostas.

Na concepção de Hurley e Hult (1998), quanto maior a presença de inovação na cultura da empresa, maior a ênfase no aprendizado, desenvolvimento de vantagem competitiva e participação na tomada de decisão. Com isso, Faleiro (2001) sugere que a orientação para aprendizagem tem relação positiva com a inovação ao difundir os novos conhecimentos extraídos do mercado de forma rápida para a organização.

O estudo de Baker e Sinkula (2007) ressalta que uma forte orientação para o mercado pode auxiliar no equilíbrio entre as inovações incrementais e radicais. Baker e Sinkula (2002) também indicam que as organizações que estão engajadas na geração de aprendizagem e no processo contínuo de inovação radical estão aptas para obter um diferencial em mercados competitivos. Pode-se dizer que a inovação radical é resultado de dois fatores: a orientação ao empreendedorismo e a orientação para o mercado de geração de informação, sendo o capital externo (redes sociais) moderador do impacto dos dois fatores, conforme analisado por Perin et al. (2009). 


\subsection{Orientação para o mercado, aprendizagem organizacional e inovação}

Day (1994), Sinkula et al. (1997) e Baker e Sinkula (1997) mencionam que a orientação para aprendizagem está relacionada positivamente com a orientação para o mercado. Nesse sentido, os autores convergem sobre a importância das organizações identificarem as necessidades e expectativas de seus clientes, aprendendo com as informações obtidas para tomada de decisão. Corroborando com essa linha de pensamento, Perin et al. (2004, p.85) mencionam que "a orientação para aprendizagem afeta indiretamente o desempenho organizacional, pelo aumento dos comportamentos orientados para o mercado, e afeta diretamente o desempenho, facilitando a aprendizagem generativa que leva à inovação dos produtos, procedimentos e sistemas".

$\mathrm{Na}$ literatura de marketing, há poucos estudos que relacionam os três construtos: orientação para o mercado, orientação para aprendizagem e inovação. Baker e Sinkula (1999, p. 299) destacam a relação proposta (Figura 1). Com base nos autores, a orientação para o mercado e a orientação para a aprendizagem interferem no desempenho da organização pelos efeitos diretos causados sobre a inovação.

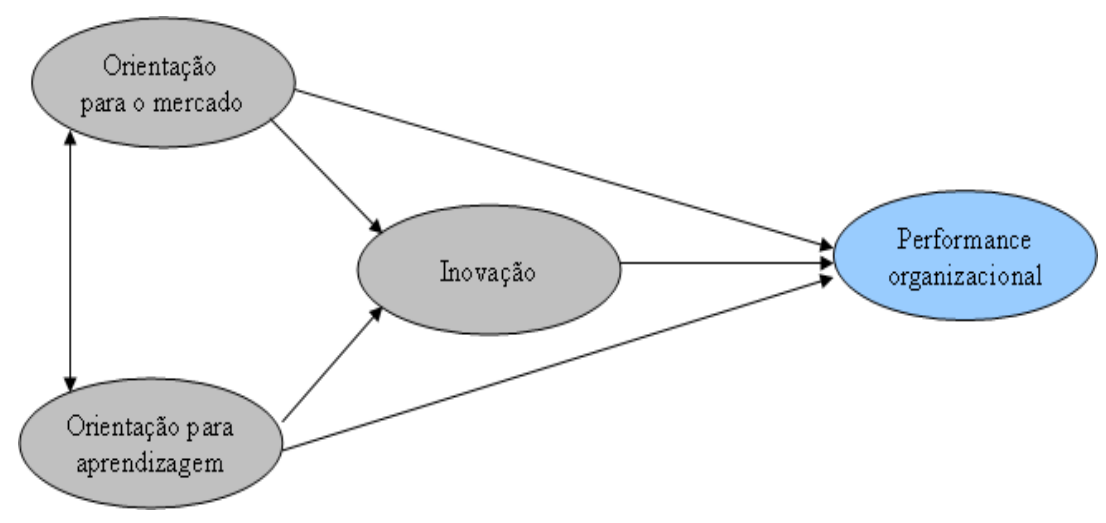

Figura 1. Efeitos da orientação para o mercado, orientação para aprendizagem e da inovação no desempenho organizacional.

Fonte: Elaborada pelos autores com base em Baker e Sinkula (1999).

As pesquisas de Perin (2001) e de Perin et al. (2004) ressaltam que a orientação para o mercado não é suficiente para gerar vantagem competitiva sustentável no longo prazo, sendo necessário considerar a aprendizagem organizacional e a inovação juntamente com a orientação para o mercado. 


\section{PROCEDIMENTOS METODOLÓGICOS}

O método de trabalho aplicado neste estudo é um survey cross-sectional. Em relação à lógica, apresenta-se o método dedutivo e, em razão de se utilizar do modelo proposto por Perin (2001), o estudo é considerado como aplicado. Em termos das técnicas adotadas, emprega-se como base inicial a pesquisa bibliográfica. Utiliza-se também a pesquisa de campo, tendo com instrumento de pesquisa a aplicação de questionários.

\subsection{População e amostra}

A população-alvo deste estudo compreendeu o conjunto de organizações de varejo associadas à Câmara de Dirigentes Lojistas de Passo Fundo. Para atender ao objetivo da pesquisa, considerou-se que das 1162 organizações de varejo associadas à CDL no mês de agosto de 2010, 3,69\% correspondem ao número de organizações de varejo enquadradas na categoria de Ópticas e Joalherias (43 organizações). O segmento de ópticas passo-fundenses foi escolhido pelo fato de apresentar um processo dinâmico de mudanças com alto índice de competitividade e por trabalhar com novas tecnologias de produtos e serviços.

Para aplicação do instrumento de coleta de dados, foram consideradas as organizações de varejo que têm como atividade principal o comércio varejista de artigos de óptica. As organizações cadastradas como comércio varejista de artigos de joalheria da categoria de "Ópticas e Joalherias" não foram consideradas para aplicação do questionário, totalizando seis organizações, conforme consulta realizada no Sistema Integrado de Informações sobre Operações Interestaduais com Mercadorias e Serviços (SINTEGRA), 2010, do Estado do Rio Grande do Sul. Na categoria, estão cadastradas ópticas que pertencem ao mesmo grupo/proprietário, sendo que na aplicação do questionário participou da pesquisa apenas uma das organizações de cada grupo. Dessa forma, não participaram da análise nove ópticas que se enquadraram nessa situação. Após expostos os cortes feitos no conjunto de 43 organizações de varejo (universo), totalizou-se o número de 28 ópticas para análise. Na forma de censo (Malhotra, 2004), as 28 organizações de varejo constituem o alvo para aplicação do instrumento de coleta de dados. 


\subsection{Instrumento e procedimento de coleta dos dados}

$\mathrm{O}$ instrumento de coleta de dados desta pesquisa foi baseado no questionário aplicado por Perin (2001), o qual considerou a análise dos três construtos: orientação para o mercado, orientação para aprendizagem, inovação e desempenho organizacional. Para operacionalização do construto de orientação para o mercado, Perin (2001) utilizou a escala Markor (Market orientation), que vem sendo utilizada em estudos brasileiros (Perin, 2001; Antoni, 2004; Perin, Sampaio e Faleiro, 2004; Perin, Sampaio, Baker e Simões, 2009) e é composta por vinte variáveis distribuídas nas dimensões geração de informações de mercado, disseminação de informações de mercado e resposta ao mercado (responsividade).

Com base em Perin (2001), para a operacionalização do construto de orientação para aprendizagem, o autor se baseou na escala desenvolvida por Sinkula et al. (1997), sendo revisada por Baker e Sinkula (1999). A escala de orientação para aprendizagem conta com 18 indicadores, os quais estão distribuídos nas dimensões comprometimento com o aprendizado, visão compartilhada e mente aberta.

Para avaliação do questionário, o respondente avalia cada variável em uma escala de seis pontos (bi-etápica), partindo da discordância total com valor de um à concordância total que corresponde ao valor seis, em que o respondente da organização se compara aos seus principais concorrentes no seu principal setor de atividade, valendo para os construtos de orientação para o mercado e para a aprendizagem.

No que diz respeito ao construto de inovação e desempenho organizacional, Perin (2001) utilizou a escala de Likert de cinco pontos, em que o respondente compara o desempenho da sua organização com o de seus principais concorrentes no período dos últimos 12 meses em seu principal setor de atividade, sendo os indicadores avaliados de um (desempenho muito menor que o dos principais concorrentes) a cinco (desempenho muito maior que o dos principais concorrentes).

Para aplicação do questionário em ópticas de Passo Fundo, foi necessária uma adaptação semântica do instrumento de coleta de dados por se tratar de organizações de pequeno e médio portes, uma vez que o questionário proposto por Perin (2001) é direcionado para aplicação em organizações de grande porte. No campo semântico, destaca-se que as expressões "empresas", "produtos ou serviços", "funcionários", "os gerentes, chefes ou líderes desta empresa", "cultura corporativa" e "número de unidades produtivas” foram substituídas por “organizações”, “serviços”, “colaboradores”, “a liderança da organização", "cultura da organização" e "número de unidades vendidas", respectivamente.

Revista de Administração e Inovação, São Paulo, v. 10, n.3, p.65-88, jul./set. 2013. 
A aplicação do questionário se deu por meio de entrevistas direcionadas para a alta administração das organizações selecionadas, nesse caso, proprietários, diretores e gerentes (Perin, 2001; Perin et al., 2004). Anteriormente à aplicação do questionário, foi enviada uma carta de apresentação da pesquisa para o e-mail do responsável da organização que constava no cadastro da CDL e feito o agendamento prévio das entrevistas por telefone. Considerando que a coleta de dados foi feita presencialmente e tendo em vista que as possíveis dúvidas que surgissem no momento da coleta seriam sanadas pelo entrevistador, não houve a necessidade de realização de pré-teste. Na ocasião das entrevistas, não foram observadas dúvidas dos entrevistados quanto aos elementos que compuseram o questionário. Com isso, julgou-se a não necessidade de alterações e adaptações no instrumento de coleta original.

Os dados foram coletados entre os dias 21 e 23 de setembro de 2010 por meio de entrevistas presenciais nas organizações de varejo analisadas. Das 28 ópticas da amostra, uma delas não foi encontrada (o número de contato não existia e o contato de e-mail retornou, em razão do que subentendese que a organização tenha encerrado suas atividades) e três optaram por não participar da pesquisa. A amostra efetiva envolveu, portanto, 24 ópticas com questionários considerados válidos, ou seja, 85,71\% do universo de organizações selecionadas para o estudo.

\subsection{Procedimento de análise e interpretação dos dados}

Os dados utilizados para análise dos resultados foram obtidos por meio do levantamento de corte transversal, considerando uma amostra efetiva de 24 ópticas. Após a coleta de dados, as informações foram processadas e analisadas por meio da modelagem de equações estruturais estimadas pelo método dos mínimos quadrados parciais (Partial Least Squares - PLS) com auxílio do software SmartPLS 2.0 M3. Adotou-se também a técnica de bootstrapping por se trabalhar com uma amostra pequena de respondentes. Essa técnica fornece as estatísticas para o teste de hipóteses de modo a validar o estudo.

Foram elaborados três modelos a partir das variáveis e escalas do estudo, sendo eles:

i) primeiro modelo: elaborado com base na escala Markor, tendo as suas três dimensões como variáveis latentes exógenas independentes; a variável inovação como variável endógena e intermediária; a variável desempenho organizacional como variável endógena e dependente;

ii) segundo modelo: construído a partir da escala de orientação para a aprendizagem, tendo as suas três dimensões como variáveis latentes exógenas independentes; a variável inovação como variável endógena e intermediária; a variável desempenho organizacional como variável endógena e dependente;

Revista de Administração e Inovação, São Paulo, v. 10, n.3, p.65-88, jul./set. 2013. 
iii) terceiro modelo: as orientações para o mercado e para a aprendizagem como variáveis latentes exógenas independentes; a variável inovação como variável endógena e intermediária; a variável desempenho organizacional como variável endógena e dependente.

Para avaliar a validade e a eficiência do modelo, foram utilizados índices obtidos com o auxílio do SmartPLS 2.0 M3 (Ringle, Wende e Will, 2005) foram utilizados. A variância extraída (AVE) representa a avaliação da validade convergente e deve apresentar valores superiores a 0,5. A confiabilidade composta deve apresentar valores superiores a 0,7 e mensura a precisão com que a variável latente mede aquilo que se propõe a medir. A confiabilidade composta, semelhante ao AVE, é calculada a partir das cargas padronizadas das variáveis latentes e dos erros obtidos na análise fatorial. $\mathrm{O}$ valor de $\mathrm{R}^{2}$ é o valor índice que indica o percentual da variância total da variável dependente e é explicado pela equação de regressão. O Alfa de Cronbach determina a validade interna do construto, sendo que se consideram satisfatórios valores acima de 0,7 (Hair, Anderson, Tatham e Black, 2005; Fornell \& Larcker, 1982).

\section{ANÁLISE DESCRITIVA}

O estudo em questão analisou a amostra efetiva de 24 ópticas passo-fundenses. A caracterização da amostra compreendeu, em sua maioria, organizações com menos de cinco anos de atividade no setor varejista e com atuação no mercado regional, as quais têm como alvo o consumidor final e possuem menos de cinco colaboradores. O principal respondente da pesquisa foi o proprietário da organização.

Para análise do resultado dos construtos e suas dimensões, consideram-se os valores de média aritmética apresentados na Tabela 1.

\begin{tabular}{ccc}
\hline Construtos e dimensões & Média & Escala de mensuração \\
\hline Geração de informações de mercado & 3,19 & Bi-etápica de 6 pontos \\
Disseminação de informações de mercado & 4,39 & Bi-etápica de 6 pontos \\
Resposta ao mercado & 3,59 & Bi-etápica de 6 pontos \\
Orientação para o mercado & $\mathbf{3 , 7 2}$ & Média dos três subconstrutos \\
Comprometimento com o aprendizado & 5,04 & Bi-etápica de 6 pontos \\
Visão compartilhada & 4,81 & Bi-etápica de 6 pontos \\
Postura de "mente aberta" & 4,57 & Bi-etápica de 6 pontos \\
Orientação para aprendizagem & $\mathbf{4 , 8 1}$ & Média dos três subconstrutos \\
Inovação & $\mathbf{3 , 3 5}$ & Likert de 5 pontos \\
Desempenho organizacional & $\mathbf{3 , 6 1}$ & Likert de 5 pontos \\
\hline
\end{tabular}

Tabela 1. Médias dos construtos orientação para o mercado, orientação para aprendizagem, inovação, desempenho organizacional e de seus subconstrutos

Fonte: Elaborada pelos autores a partir dos dados da pesquisa, 2011. 
Com base nos resultados do construto de orientação para o mercado, infere-se que as ópticas estudadas têm uma abordagem internalizada para definição de suas estratégias e ações. Nesse contexto, ações mais voltadas para o mercado são sugeridas, como uma forma de aproximação do contexto externo no qual as ópticas estão inseridas, considerando informações do mercado para identificar necessidades e expectativas atuais e futuras de seus clientes.

Os graus de orientação para o mercado e para a aprendizagem das organizações podem ser melhorados, fomentando a geração e a disseminação de informações de mercado e de resposta a este, bem como o comprometimento com a aprendizagem, visão compartilhada entre os membros da organização em todos os níveis hierárquicos e a postura de mente aberta. Dessa forma, pode haver um maior efeito direto dos construtos abordados anteriormente na inovação que, por sua vez, implicará um desempenho organizacional superior para as ópticas passo-fundenses, conforme a abordagem proposta por Baker e Sinkula (1999).

\section{MODELAGEM DE EQUAÇÕES ESTRUTURAIS}

A partir da percepção dos 24 respondentes das ópticas estudadas, modelos baseados em equações estruturais foram elaborados, utilizando-se o método de estimação de mínimos quadrados parciais (PLS). O primeiro modelo elaborado e analisado possui as três dimensões da escala Markor como variáveis latentes exógenas e independentes; a variável inovação como variável endógena e intermediária; a variável desempenho organizacional como variável endógena e dependente. O primeiro modelo pode ser observado na Figura 2, com os coeficientes de caminhos (path coefficients) entre as variáveis latentes. 


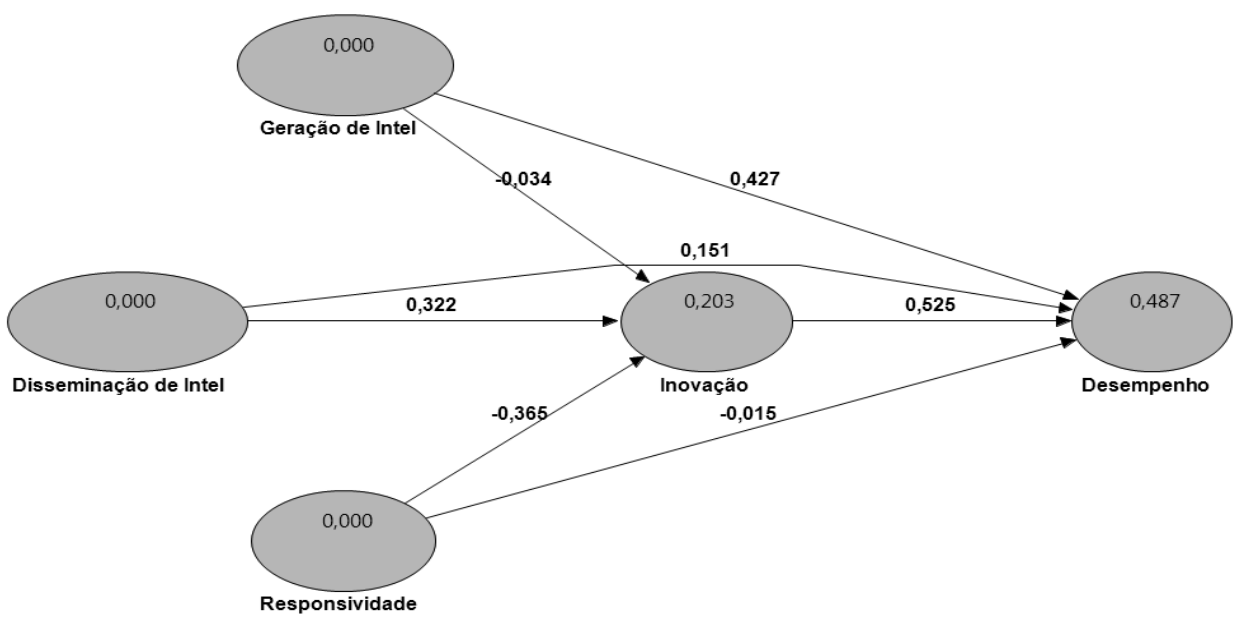

Figura 2. Primeiro modelo estrutural com PLS e coeficientes de caminhos

Fonte: Elaborada pelos autores a partir dos dados da pesquisa, 2011.

A partir dos coeficientes de caminhos apresentados na Figura 2, verifica-se que a relação entre a inovação e o desempenho é elevada. Observa-se também que a geração de inteligência afeta moderadamente o desempenho, assim como a disseminação de inteligência e a responsividade afetam de forma moderada a inovação, mesmo a responsividade afetando de modo negativo. $\mathrm{Na}$ Tabela 2, são apresentados os valores de AVE, confiabilidade composta, $\mathrm{R}^{2}$ e o Alfa de Cronbach das variáveis latentes do primeiro modelo.

\begin{tabular}{ccccc} 
& AVE & Confiabilidade composta & $\mathbf{R}^{2}$ & Alfa de Cronbach \\
\hline Desempenho & 0,677 & 0,862 & 0,487 & 0,763 \\
Disseminação de inteligência & 0,476 & 0,702 & & 0,647 \\
Geração de inteligência & 0,535 & 0,820 & & 0,726 \\
Inovação & 0,698 & 0,874 & 0,203 & 0,784 \\
Responsividade & 0,374 & 0,813 & & 0,794 \\
\hline
\end{tabular}

Tabela 2. Índices de avaliação das variáveis latentes do primeiro modelo

Fonte: Elaborada pelos autores a partir dos dados da pesquisa, 2011.

Observa-se que os valores de AVE são satisfatórios para as variáveis desempenho, geração de inteligência e inovação. Não foram eliminados os indicadores que apresentaram cargas fatoriais insatisfatórias, de modo a manter todos os indicadores da escala original (Markor). Os valores de confiabilidade composta foram todos maiores que 0,7 , sendo todos considerados satisfatórios. Os valores de $\mathrm{R}^{2}$ foram considerados medianamente satisfatórios, ressaltando que o resultado da variável desempenho obteve resultado muito superior ao da inovação. Os valores dos Alfas de Cronbach foram todos acima de 0,7, considerados satisfatórios. Contudo, cabe ressaltar a exceção do alfa da disseminação de inteligência $(0,647)$. 
As correlações entre as variáveis latentes do primeiro modelo foram analisadas e os valores podem ser observados na Tabela 3. Na diagonal principal, estão os valores da raiz quadrada do AVE (em negrito).

\begin{tabular}{|c|c|c|c|c|c|}
\hline & Desempenho & $\begin{array}{c}\text { Disseminação de } \\
\text { inteligência }\end{array}$ & $\begin{array}{l}\text { Geração de } \\
\text { inteligência }\end{array}$ & Inovação & Responsividade \\
\hline Desempenho & $\mathbf{0 , 8 2 3}$ & & & & \\
\hline Disseminação de inteligência & $\begin{array}{c}0,353 \\
\mathrm{p}=0,090\end{array}$ & $\mathbf{0 , 6 9 0}$ & & & \\
\hline Geração de inteligência & $\begin{array}{c}0,382 \\
\mathrm{p}=0,066\end{array}$ & $\begin{array}{c}0,171 \\
\mathrm{p}=0,424\end{array}$ & $\mathbf{0 , 7 3 2}$ & & \\
\hline Inovação & $\begin{array}{c}0,515^{*} \\
\mathrm{p}=0,010\end{array}$ & $\begin{array}{c}0,252 \\
\mathrm{p}=0,235\end{array}$ & $\begin{array}{c}-0,124 \\
\mathrm{p}=0,562\end{array}$ & $\mathbf{0 , 8 3 5}$ & \\
\hline Responsividade & $\begin{array}{c}0,012 \\
\mathrm{p}=0,955\end{array}$ & $\begin{array}{c}0,176 \\
\mathrm{p}=0,411\end{array}$ & $\begin{array}{c}0,398 \\
\mathrm{p}=0,054\end{array}$ & $\begin{array}{c}-0,322 \\
\mathrm{p}=0,125\end{array}$ & 0,612 \\
\hline
\end{tabular}

Tabela 3. Correlações, significância e raiz quadrada do AVE das variáveis do primeiro modelo

Fonte: Elaborada pelos autores a partir dos dados da pesquisa, 2011.

Nota.* Significativo ao nível de $\mathrm{p}<0,05 ; * *$ Significativo ao nível de $\mathrm{p}<0,01 ; * * *$ Significativo ao nível de $\mathrm{p}<0,001$.

As correlações obtidas entre as variáveis latentes do primeiro modelo não apresentaram significância, com exceção da correlação entre a inovação e o desempenho. Assim, constata-se inicialmente que as variáveis não são satisfatoriamente interdependentes. Em contrapartida, pode-se afirmar que o modelo apresenta uma baixa multicolinearidade, o que pode ser considerado como positivo para o modelo em questão.

Utilizou-se a técnica de bootstrapping (programada para 100 casos e 200 amostras). Obtiveram-se os valores da estatística $\mathrm{T}$ para as relações entre as variáveis latentes do primeiro modelo. Os valores obtidos por meio da aplicação da técnica e seus efeitos totais no modelo são apresentados na Tabela 4. Também é apresentada a situação das hipóteses do modelo, sendo aceitas no caso de pesos significativamente diferentes de zero $(\mathrm{p}<0,05$ - bicaudal).

\begin{tabular}{|c|c|c|c|c|c|}
\hline & $\begin{array}{c}\text { Path Coefficient } \\
\text { original }\end{array}$ & $\begin{array}{c}\text { Coeficiente após o } \\
\text { Bootstrapping }\end{array}$ & $\begin{array}{c}\text { Erro } \\
\text { padrão }\end{array}$ & $\begin{array}{c}\text { Estatística } \\
\mathbf{T}\end{array}$ & Hipóteses \\
\hline $\begin{array}{l}\text { Disseminação de inteligência - } \\
\text { desempenho }\end{array}$ & 0,328 & 0,325 & 0,129 & $2,551^{*}$ & $\begin{array}{c}\text { Aceita } \\
p=0,011\end{array}$ \\
\hline $\begin{array}{l}\text { Disseminação de inteligência - } \\
\text { inovação }\end{array}$ & 0,277 & 0,280 & 0,146 & 1,895 & $\begin{array}{l}\text { Rejeita } \\
\mathrm{p}=0,060\end{array}$ \\
\hline $\begin{array}{l}\text { Geração de inteligência - } \\
\text { desempenho }\end{array}$ & 0,404 & 0,401 & 0,079 & $5,109 * * *$ & $\begin{array}{l}\text { Aceita } \\
\mathrm{p}=, 000\end{array}$ \\
\hline $\begin{array}{l}\text { Geração de inteligência - } \\
\text { inovação }\end{array}$ & $-0,054$ & $-0,047$ & 0,105 & 0,514 & $\begin{array}{l}\text { Rejeita } \\
\mathrm{p}=0,608\end{array}$ \\
\hline Inovação - desempenho & 0,501 & 0,502 & 0,092 & $5,432 * * *$ & $\begin{array}{l}\text { Aceita } \\
\mathrm{p}=0,000\end{array}$ \\
\hline Responsividade - desempenho & $-0,118$ & $-0,125$ & 0,141 & 0,838 & $\begin{array}{c}\text { Rejeita } \\
\mathrm{p}=0,403\end{array}$ \\
\hline $\begin{array}{l}\text { Responsividade - } \\
\text { Inovaçãoinovação }\end{array}$ & $-0,342$ & $-0,347$ & 0,151 & $2,259^{*}$ & $\begin{array}{l}\text { Aceita } \\
\mathrm{p}=0,025\end{array}$ \\
\hline
\end{tabular}

Tabela 4. Estatísticas $\mathbf{T}$ e teste de hipóteses do primeiro modelo

Revista de Administração e Inovação, São Paulo, v. 10, n.3, p.65-88, jul./set. 2013. 
Fonte: Elaborada pelos autores a partir dos dados da pesquisa, 2011.

Nota.* Significativo ao nível de $\mathrm{p}<0,05$; ** Significativo ao nível de $\mathrm{p}<0,01 ; * * *$ Significativo ao nível de $\mathrm{p}<0,001$.

A partir dos resultados dos testes de hipóteses apresentados na Tabela 4, afirma-se que a disseminação de inteligência e a geração de inteligência das organizações estudadas afetam positiva e significativamente o desempenho. No entanto, infere-se que as ações voltadas para a construção de inteligência estratégica geram condições competitivas para as organizações estudadas. Destaca-se que a inovação afeta positiva e significativamente o desempenho das ópticas. Entretanto, o inverso é válido ao considerar a relação entre as variáveis responsividade e inovação, o que contradiz os pressupostos teóricos e lógicos. Considerando que a responsividade é uma postura prática de aplicação estratégica da inteligência gerada e disseminada, a evidência de relação negativa entre a responsividade e a inovação sugere que as ações estratégicas das ópticas pesquisadas podem não estar voltadas para a inovação, entretanto, possivelmente para questões relacionadas ao mercado e aos seus clientes.

Nesse contexto, pode-se afirmar que quanto maior o nível de responsividade na adoção de inteligência de mercado para fins estratégicos, menor é o nível de inovação das ópticas em análise. As demais relações não foram suportadas estatisticamente. A geração de inteligência não afeta significativamente a inovação; somente quando a inteligência é disseminada é que esse efeito se torna significativo. Dessa forma, expõe-se que a geração sem a disseminação de inteligência não apresenta impactos significantes na inovação.

O segundo modelo elaborado e analisado apresenta as três dimensões da escala de orientação para a aprendizagem como variáveis latentes exógenas independentes; a variável inovação como variável endógena e intermediária; a variável desempenho organizacional como variável endógena e dependente.

$\mathrm{Na}$ Figura 3, podem ser observados os coeficientes de caminhos (path coefficients) entre as variáveis latentes.

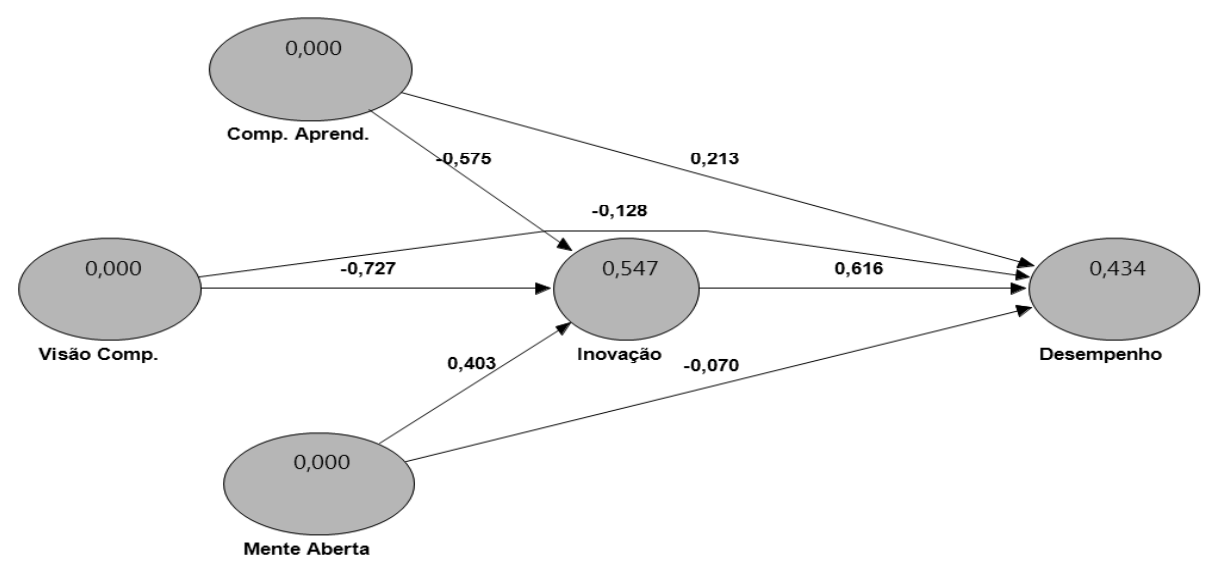

Revista de Administração e Inovação, São Paulo, v. 10, n.3, p.65-88, jul./set. 2013. 


\section{Figura 3. Segundo modelo estrutural com PLS e coeficientes de caminhos}

Fonte: Elaborada pelos autores a partir dos dados da pesquisa, 2011.

O diagrama de caminhos da Figura 3 permite observar que existe uma relação positiva e elevada entre as variáveis mente aberta e inovação, bem como entre a inovação e o desempenho. Entretanto, o diagrama apresenta também relações fortes e negativas, destacando as relações entre a visão compartilhada e a inovação e entre o comprometimento com a aprendizagem e a inovação. As constatações iniciais divergem de alguns pressupostos teóricos e empíricos, com base nos quais se esperaria encontrar relações de interdependência positivas entre os construtos de aprendizagem e de inovação.

Na sequência da análise do segundo modelo, considera-se a Tabela 5, a qual contempla os valores de AVE, confiabilidade composta, $\mathrm{R}^{2}$ e o Alfa de Cronbach das variáveis do modelo.

\begin{tabular}{ccccc}
\cline { 2 - 5 } & AVE & $\begin{array}{c}\text { Confiabilidade } \\
\text { composta }\end{array}$ & $\mathbf{R}^{\mathbf{2}}$ & $\begin{array}{c}\text { Alfa de } \\
\text { Cronbach }\end{array}$ \\
\hline $\begin{array}{c}\text { Comprometimento com a } \\
\text { aprendizagem }\end{array}$ & 0,419 & 0,644 & & 0,763 \\
Desempenho & 0,632 & 0,835 & 0,434 & 0,763 \\
Inovação & 0,700 & 0,875 & 0,547 & 0,784 \\
Mente aberta & 0,505 & 0,854 & & 0,823 \\
Visão compartilhada & 0,215 & 0,470 & & 0,754 \\
\hline
\end{tabular}

Tabela 5. Índices de avaliação das variáveis do segundo modelo

Fonte: Elaborada pelos autores a partir dos dados da pesquisa, 2011.

Destaca-se que os valores de AVE e de confiabilidade composta do comprometimento com a aprendizagem e da visão compartilhada não foram satisfatórios. Foram mantidos todos os itens das dimensões da escala original, mesmo apresentando cargas fatoriais baixas. Os demais construtos apresentaram valores de AVE e confiabilidade composta satisfatórios. Já os $\mathrm{R}^{2}$ foram positivos, apresentando uma alta variação explicada pelas variáveis independentes. Os valores de Alfa de Cronbach apresentaram alta pertinência, ou seja, todos se mostraram maiores que 0,7 .

Da mesma forma que no modelo anterior, foram analisadas as correlações entre as variáveis latentes do segundo modelo. Na Tabela 6, os índices de correlação são observados entre as variáveis latentes do segundo modelo. Na diagonal principal, estão os valores da raiz quadrada do AVE.

\begin{tabular}{cccccc}
\cline { 2 - 5 } & $\begin{array}{c}\text { Comprometimento com } \\
\text { a aprendizagem }\end{array}$ & Desempenho & Inovação & $\begin{array}{c}\text { Mente } \\
\text { aberta }\end{array}$ & $\begin{array}{c}\text { Visão } \\
\text { compartilhada }\end{array}$ \\
\hline $\begin{array}{c}\text { Compromentimento com } \\
\text { a aprendizagem }\end{array}$ & $\mathbf{0 , 6 4 7}$ & & & & \\
Desempenho & $-0,165$ & $\mathbf{0 , 7 9 5}$ & & \\
Inovação & $\mathrm{p}=0,442$ & $0,624^{* *}$ & $\mathbf{0 , 8 3 7}$ & & \\
& $-0,482^{*}$ & &
\end{tabular}

Revista de Administração e Inovação, São Paulo, v. 10, n.3, p.65-88, jul./set. 2013. 


\begin{tabular}{cccccc} 
& $\mathrm{p}=0,017$ & $\mathrm{p}=0,001$ & & & \\
Mente aberta & $0,686^{* * *}$ & $-0,281$ & $-0,448^{*}$ & $\mathbf{0 , 7 1 1}$ & \\
& $\mathrm{p}=0,000$ & $\mathrm{p}=0,184$ & $\mathrm{p}=0,028$ & & \\
Visão compartilhada & 0,252 & $-0,500^{*}$ & $-0,619^{* *}$ & $0,628^{* *}$ & $\mathbf{0 , 4 6 3}$ \\
\hline
\end{tabular}

Tabela 6. Correlações, significância e raiz quadrada do AVE das variáveis do segundo modelo

Fonte: Elaborada pelos autores a partir dos dados da pesquisa, 2011.

Nota.* Significativo ao nível de $\mathrm{p}<0,05$; ** Significativo ao nível de $\mathrm{p}<0,01 ; * * *$ Significativo ao nível de $\mathrm{p}<0,001$.

Observando os valores de correlação e as suas significâncias na Tabela 6, percebe-se que há correlações significativas no segundo modelo. Constatou-se resultado significativo e negativo entre as relações: inovação e comprometimento com a aprendizagem, mente aberta e inovação, visão compartilhada e desempenho. Assim, com base na visão dos participantes do estudo, afirma-se que o aumento dos níveis de comprometimento com a aprendizagem e de mente aberta dos membros das organizações resulta em uma diminuição do nível de inovação.

Um aumento na visão compartilhada dos membros resulta na diminuição do desempenho das ópticas, na concepção dos gestores participantes do estudo. Por outro lado, constata-se a existência de altas correlações significativas e positivas entre: comprometimento com a aprendizagem e mente aberta, visão compartilhada e mente aberta, inovação e desempenho. Então, pode-se afirmar que essas variáveis possuem uma interdependência fortemente positiva.

Para análise do segundo modelo, foi utilizada a técnica de bootstrapping (programada para 100 casos e 200 amostras). Os valores obtidos por meio da aplicação da técnica e seus efeitos totais no modelo são apresentados na Tabela 7. A situação das hipóteses do segundo modelo também são destacadas, sendo aceitas se apresentarem pesos significativamente diferentes de zero ( $\mathrm{p}<0,05$ - bicaudal).

\begin{tabular}{|c|c|c|c|c|c|}
\hline & $\begin{array}{c}\text { Path Coefficient } \\
\text { original }\end{array}$ & $\begin{array}{c}\text { Path Coefficient após } \\
\text { Bootstrapping }\end{array}$ & $\begin{array}{l}\text { Erro } \\
\text { padrão }\end{array}$ & $\begin{array}{c}\text { Estatística } \\
T\end{array}$ & Hipótese \\
\hline $\begin{array}{l}\text { Comprometimento com a } \\
\text { aprendizagem - desempenho }\end{array}$ & 0,043 & 0,155 & 0,233 & 0,185 & $\begin{array}{c}\text { Rejeita } \\
\mathrm{p}=0,854\end{array}$ \\
\hline $\begin{array}{l}\text { Comprometimento com a } \\
\text { aprendizagem - inovação }\end{array}$ & $-0,250$ & $-0,112$ & 0,260 & 0,961 & $\begin{array}{c}\text { Rejeita } \\
\mathrm{p}=0,337\end{array}$ \\
\hline Inovação - desempenho & 0,581 & 0,549 & 0,099 & $5,846 * * *$ & $\begin{array}{c}\text { Aceita } \\
\mathrm{p}=0,000\end{array}$ \\
\hline Mente aberta - desempenho & 0,051 & $-0,029$ & 0,212 & 0,239 & $\begin{array}{c}\text { Rejeita } \\
\mathrm{p}=0,811\end{array}$ \\
\hline Mente aberta - inovação & $-0,121$ & $-0,203$ & 0,181 & 0,669 & $\begin{array}{c}\text { Rejeita } \\
\mathrm{p}=0,504\end{array}$ \\
\hline $\begin{array}{l}\text { Visão compartilhada - } \\
\text { desempenho }\end{array}$ & $-0,378$ & $-0,352$ & 0,120 & $3,164 * *$ & $\begin{array}{c}\text { Aceita } \\
\mathrm{p}=0,002\end{array}$ \\
\hline $\begin{array}{l}\text { Visão compartilhada - } \\
\text { inovação }\end{array}$ & $-0,252$ & $-0,245$ & 0,113 & $2,234 *$ & $\begin{array}{c}\text { Aceita } \\
\mathrm{p}=0,027\end{array}$ \\
\hline
\end{tabular}

Tabela 7. Estatísticas $\mathbf{T}$ e teste de hipóteses do segundo modelo

Fonte: Elaborada pelos autores a partir dos dados da pesquisa, 2011.

Nota.* Significativo ao nível de $\mathrm{p}<0,05 ; * *$ Significativo ao nível de $\mathrm{p}<0,01 ; * * *$ Significativo ao nível de $\mathrm{p}<0,001$.

Revista de Administração e Inovação, São Paulo, v. 10, n.3, p.65-88, jul./set. 2013. 
De acordo com a Tabela 7, evidencia-se que a inovação e a visão compartilhada afetam significativamente o desempenho, que, por sua vez, é positivamente afetado pela inovação e negativamente pela visão compartilhada. Verifica-se que a visão compartilhada afeta negativa e significativamente a inovação. Na visão dos respondentes, as influências negativas sugerem que a visão compartilhada dos membros das organizações prejudica os aspectos estratégicos organizacionais. Por se tratar de uma pesquisa com organizações de pequeno porte, essa evidência sugere que a estrutura, possivelmente centralizada, das ópticas pesquisadas, não considera a visão compartilhada de seus membros como um elemento positivo. Com isso, a inovação pode ser fruto de decisões e ações concentradas no nível hierárquico mais elevado. As demais hipóteses foram rejeitadas por não apresentarem significância estatística.

As orientações para o mercado e para a aprendizagem como variáveis latentes exógenas independentes estão inseridas no terceiro modelo elaborado e testado, bem como a variável inovação como endógena e intermediária e a variável desempenho organizacional como variável endógena e dependente. O modelo pode ser observado na Figura 4, a qual contém os coeficientes de caminhos entre as variáveis latentes.

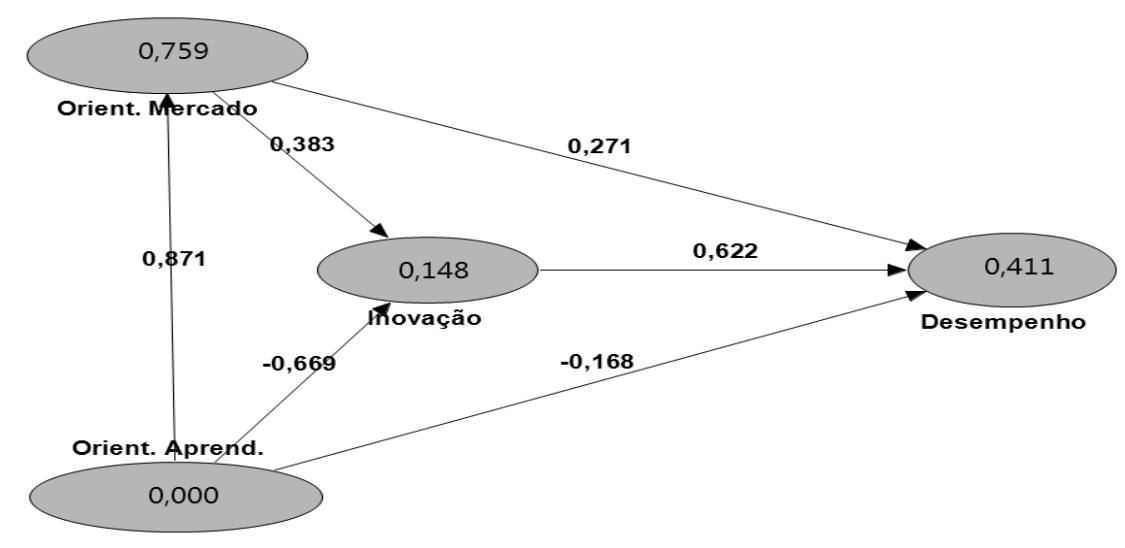

Figura 4. Terceiro modelo estrutural com PLS e coeficientes de caminhos

Fonte: Elaborada pelos autores a partir dos dados da pesquisa, 2011.

No modelo de caminhos da Figura 4, há uma relação alta entre as orientações para a aprendizagem e para o mercado. Constata-se que a orientação para a aprendizagem apresenta uma relação negativa e considerável com a inovação. A orientação para o mercado demonstra ter um coeficiente de influência positivo e mediano sobre a inovação. Essas relações são confirmadas estatisticamente com o teste de hipóteses.

Revista de Administração e Inovação, São Paulo, v. 10, n.3, p.65-88, jul./set. 2013. 
Em relação ao terceiro modelo, são apresentados, na Tabela 8, os valores de AVE, confiabilidade composta, $\mathrm{R}^{2}$ e o Alfa de Cronbach dos construtos.

\begin{tabular}{ccccc}
\cline { 2 - 5 } & AVE & $\begin{array}{c}\text { Confiabilidade } \\
\text { composta }\end{array}$ & $\mathbf{R}^{\mathbf{2}}$ & $\begin{array}{c}\text { Alfa de } \\
\text { Cronbach }\end{array}$ \\
\hline Desempenho & 0,634 & 0,836 & 0,411 & 0,763 \\
Inovação & 0,699 & 0,874 & 0,148 & 0,784 \\
Orientação para aprendizagem & 0,387 & 0,904 & - & 0,885 \\
Orientação para mercado & 0,312 & 0,858 & 0,759 & 0,824 \\
\hline
\end{tabular}

Tabela 8. Índices de avaliação dos construtos do terceiro modeloFonte: Elaborada pelos autores a partir dos dados da pesquisa, 2011.

A partir da análise da Tabela 8, evidencia-se que as orientações para a aprendizagem e para o mercado apresentaram um baixo AVE, o que pode prejudicar as relações do modelo e as constatações do estudo. Porém, todos os itens das escalas foram mantidos de modo a trabalhar com os construtos originais e completos. Os valores de confiabilidade composta e os Alfas de Cronbach mostraram-se todos satisfatórios (acima de 0,7). Os valores de $\mathrm{R}^{2}$ foram considerados positivos, sendo que apenas o $\mathrm{R}^{2}$ da variável inovação apresenta limitações por ser muito baixo, mesmo considerando as ciências sociais.

No terceiro modelo, foram analisadas as correlações entre os construtos do estudo. Na Tabela 9, estão calculados os índices de correlação entre as variáveis latentes. Na diagonal principal, encontram-se os valores da raiz quadrada do AVE.

\begin{tabular}{ccccc}
\cline { 2 - 5 } & Desempenho & Inovação & $\begin{array}{c}\text { Orientação para } \\
\text { aprendizagem }\end{array}$ & $\begin{array}{c}\text { Orientação } \\
\text { para mercado }\end{array}$ \\
\hline Desempenho & $\mathbf{0 , 7 9 6}$ & & & \\
Inovação & $0,624 * *$ & $\mathbf{0 , 8 3 6}$ & & \\
& $\mathrm{p}=0,001$ & $-0,335$ & & \\
Orientação para aprendizagem & $-0,140$ & $-0,622$ & \\
Orientação para mercado & $\mathrm{p}=0,514$ & $\mathrm{p}=0,110$ & $\mathbf{0 , 6 2 2}$ & \\
& 0,001 & $-0,199$ & $0,871^{* * *}$ & $\mathbf{0 , 5 5 9}$ \\
\hline
\end{tabular}

Tabela 9. Correlações, significância e raiz quadrada do AVE dos construtos do terceiro modelo Fonte: Elaborada pelos autores a partir dos dados da pesquisa, 2011.

Nota.* Significativo ao nível de $\mathrm{p}<0,05 ; * *$ Significativo ao nível de $\mathrm{p}<0,01 ; * * *$ Significativo ao nível de $\mathrm{p}<0,001$.

Baseando-se na Tabela 9, as correlações significativas foram observadas entre a orientação para o mercado e a orientação para a aprendizagem, como também entre a inovação e o desempenho. O fato de as duas orientações apresentarem uma elevada correlação positiva permite afirmar que ambas podem ser encaradas como interdependentes e possivelmente sinérgicas; da mesma forma, que a inovação e o desempenho são, neste estudo, vistos como interdependentes.

No último modelo proposto, foi adotada a técnica de bootstrapping (programada para 100 casos e 200 amostras). Obtiveram-se os valores de T para as relações entre os construtos do terceiro modelo. Os

Revista de Administração e Inovação, São Paulo, v. 10, n.3, p.65-88, jul./set. 2013. 
valores obtidos por meio da aplicação da técnica e seus efeitos totais no modelo são destacados na Tabela 10, em que se apresenta também a situação das hipóteses do modelo, sendo aceitas no caso de pesos significativamente diferentes de zero $(\mathrm{p}<0,05$ - bicaudal $)$.

\begin{tabular}{|c|c|c|c|c|c|}
\hline & $\begin{array}{c}\text { Path Coefficient } \\
\text { original }\end{array}$ & $\begin{array}{c}\text { Path Coefficient } \\
\text { após Bootstrapping }\end{array}$ & $\begin{array}{l}\text { Erro } \\
\text { padrão }\end{array}$ & $\begin{array}{c}\text { Estatística } \\
\mathbf{T}\end{array}$ & Hipóteses \\
\hline Inovação - desempenho & 0,511 & 0,520 & 0,094 & $5,440 * * *$ & $\begin{array}{c}\text { Aceita } \\
\mathrm{p}=0,000\end{array}$ \\
\hline $\begin{array}{l}\text { Orientação para aprendizagem } \\
\text { - desempenho }\end{array}$ & $-0,101$ & $-0,056$ & 0,161 & 0,628 & $\begin{array}{l}\text { Rejeita } \\
\mathrm{p}=0,531\end{array}$ \\
\hline $\begin{array}{l}\text { Orientação para aprendizagem } \\
\text { - inovação }\end{array}$ & $-0,360$ & $-0,342$ & 0,130 & $2,761 * *$ & $\begin{array}{c}\text { Aceita } \\
\mathrm{p}=0,006\end{array}$ \\
\hline $\begin{array}{l}\text { Orientação para aprendizagem } \\
\text { - orientação para mercado }\end{array}$ & 0,840 & 0,845 & 0,038 & $22,139 * * *$ & $\begin{array}{c}\text { Aceita } \\
\mathrm{p}=0,000\end{array}$ \\
\hline $\begin{array}{l}\text { Orientação para mercado - } \\
\text { desempenho }\end{array}$ & 0,725 & 0,679 & 0,277 & $2,614 *$ & $\begin{array}{l}\text { Aceita } \\
\mathrm{p}=0,010\end{array}$ \\
\hline $\begin{array}{l}\text { Orientação para mercado - } \\
\text { inovação }\end{array}$ & 0,338 & 0,347 & 0,242 & 1,397 & $\begin{array}{c}\text { Rejeita } \\
p=0,164\end{array}$ \\
\hline
\end{tabular}

Tabela 10. Estatísticas T e teste de hipóteses do terceiro modeloFonte: Elaborada pelos autores a partir dos dados da pesquisa, 2011.

Nota.* Significativo ao nível de $\mathrm{p}<0,05$; ** Significativo ao nível de $\mathrm{p}<0,01 ; * * *$ Significativo ao nível de $\mathrm{p}<0,001$.

No teste de hipóteses do terceiro modelo (Tabela 10), constata-se que a orientação para o mercado afeta significativa e positivamente o desempenho das organizações participantes da pesquisa. Infere-se que a orientação para a aprendizagem aumenta consideravelmente a orientação para o mercado, porém, diminui a inovação. Essa última constatação se aproxima dos resultados obtidos no estudo semelhante desenvolvido por Baker e Sinkula (1999) e corrobora com as evidências do estudo de Perin et al. (2004). Assim, os resultados também sugerem que a orientação para a aprendizagem deva ser encarada como um antecedente da orientação para o mercado (Abbade, Zanini e Souza, 2012). Também é possível perceber que a inovação eleva significativamente o desempenho das organizações que aderiram ao estudo. As demais relações não puderam ser confirmadas estatisticamente.

\section{CONSIDERAÇÕES FINAIS}

A revisão de literatura indicou uma contribuição significativa de estudos que retratam as relações entre orientação para o mercado, orientação para aprendizagem e inovação no desempenho organizacional. Destaca-se que o tema em questão é de suma importância para o desenvolvimento de estudos no âmbito organizacional, principalmente no que se refere ao setor de serviços e ao contexto brasileiro. 
A literatura de marketing supracitada evidenciou que a orientação para o mercado reflete positivamente no desempenho das organizações, uma vez que gera uma vantagem competitiva no longo prazo. A combinação entre a orientação para o mercado e a aprendizagem reflete em efeitos sobre a inovação de produtos e serviços, o que pode acarretar num desempenho superior para as organizações.

Diante disso, decidiu-se avaliar o grau de orientação para o mercado, a aprendizagem organizacional e a inovação para o contexto de ópticas passo-fundenses associadas à Câmara de Dirigentes Lojistas da cidade de PassoFundo/RS. A escolha dessa categoria de organizações de varejo se deu pelo fato de elas apresentarem um processo dinâmico de mudanças e por serem organizações que trabalham com novas tecnologias de produtos e serviços.

Com esse intuito, o instrumento de pesquisa para a coleta dos dados, bem como a análise das informações, foram embasados no estudo de Perin (2001). A análise dos dados foi feita por meio da análise descritiva simples e de técnicas de análise multivariada para a elaboração de equação estrutural. Por se trabalhar com uma amostra pequena, o método de estimação para a modelagem de equações foi o dos mínimos quadrados parciais (PLS), associado à utilização da técnica de bootstrapping.

Os resultados obtidos com a análise do setor de atividade contribuíram para determinar o grau dos construtos e subconstrutos descritos neste trabalho. Com base nos resultados do construto de orientação para o mercado, infere-se que as ópticas estudadas têm uma abordagem internalizada para definição de estratégias e ações. Os graus de orientação para o mercado e de orientação para aprendizagem das organizações podem ser melhorados, fomentando a geração e disseminação de informações de mercado e de resposta a este, bem como o comprometimento com a aprendizagem, a visão compartilhada entre os membros da organização em todos os níveis hierárquicos e a postura de mente aberta. Dessa forma, pode haver um maior efeito direto dos construtos abordados anteriormente na inovação que, por sua vez, implicará um desempenho organizacional superior para as ópticas passo-fundenses, conforme a abordagem de Baker e Sinkula (1999).

Por meio da construção dos modelos estruturais estimados pelo método dos mínimos quadrados parciais (PLS) e com o emprego da técnica bootstrapping, constatou-se que a disseminação de inteligência, a geração de inteligência e a inovação afetam positiva e significativamente o desempenho das organizações analisadas, confirmando assim as hipóteses do estudo. Entretanto, um resultado não esperado foi a observância de que a responsividade afeta significativamente e de modo negativo a inovação das organizações. Outro ponto que merece atenção é o fato de que a visão compartilhada dos membros da organização afeta significativa e negativamente o desempenho e a inovação das ópticas 
estudadas. Portanto, julgam-se necessárias investigações adicionais que possam esclarecer e explicar o porquê da relação inversa observada.

Finalmente, o estudo confirma que a inovação e a orientação para o mercado elevam significativamente o desempenho das organizações, corroborando com evidências já reportadas por outros autores. No entanto, a orientação para a aprendizagem exerce uma influência negativa e significativa no nível inovador das organizações, mesmo influenciando positivamente e de forma altamente significativa a orientação para o mercado. Essa evidência sugere a necessidade de estudos adicionais que venham a explorar com maior propriedade tal relação negativa.

Como limitações enfrentadas na pesquisa, pode-se destacar os itens: i) os o instrumento de coleta de dados foi respondido por um membro da organização, sendo importante o comparativo de respostas múltiplas, ou seja, por mais pessoas da mesma organização; ii) as respostas sobre inovação foram coletadas com base em percepções dos entrevistados e não em fatos, o que traz uma conotação subjetiva; iii) a coleta de dados foi realizada somente por um meio de captação de informações (questionário).

As contribuições deste estudo e suas limitações oferecem uma base para estudos teóricos e empíricos sobre o tema em questão. Sugere-se também o desenvolvimento de estudos na mesma linha aplicada nesta pesquisa e análises complementares sugeridas anteriormente para toda a população de organizações associada à Câmara de Dirigentes Lojistas de Passo Fundo.

\section{REFERÊNCIAS}

Abbade, E. B., Zanini, R. R., \& Souza, A. M. (2012). Orientação para aprendizagem, orientação para mercado e desempenho organizacional: evidências empíricas. Revista de Administração Contemporânea, 16(1), 118-136.

Antoni, V. L. (2004). A relação entre orientação para o mercado e performance organizacional: um estudo nos cursos de bacharelado em administração da região sul do Brasil (Tese de doutorado). Universidade Federal de Santa Catarina, Florianópolis, SC, Brasil.

Baker, W. E., \& Sinkula, J. M. (1999). Learning orientation, market orientation, and innovation: integrating and extending models of organizational performance. Journal of Market Focused Management, 4(4), 295-308.

Baker, W. E., \& Sinkula, J. M. (2002). Market orientation, learning orientation and product innovation: delving into the Organization's Black Box. Journal of Market-Focused Management, 5(1), 5-23.

Baker, W. E., \& Sinkula, J. M. (2007). Does market orientation facilitate balanced innovation programs? An organizational learning perspective. Journal of Product Innovation Management, 24(4), 316-334.

Revista de Administração e Inovação, São Paulo, v. 10, n.3, p.65-88, jul./set. 2013. 
Câmara de Dirigentes Lojistas de Passo Fundo. (2010). Banco de dados. Passo Fundo, RS, Brasil.

Day, G. S. (1994a). Continuos learning about markets. California Management Review, 36(4), 9-31.

Day, G. S. (1994b). The capabilities of market-driven organization. Journal of Marketing, 58(4), 37-52.

Drucker, P. F. (1998). The discipline of innovation. Harvard Business Review, 1-8.

Erdil, S., Erdil, O., \& Keskin, H. (2003). The relationships between market orientation, firm innovativeness and innovation performance. Journal of Global Business and Technology, 1, 1-11.

Faleiro, S. N. (2001). A relação entre orientação para o mercado, orientação para aprendizagem $e$ inovação: o caso dos cursos de graduação em administração filiados à Angrad (Dissertação de mestrado). Universidade Federal do Rio Grande do Sul, Porto Alegre, RS, Brasil.

Fornell, C., \& Larcker, D. (1982). Evaluating structural equation models with unobservable variables and measurement error. Journal of Marketing Research, 17(1), 39-50.

Hair, J. F., Anderson, R. E., Tatham, R. L., \& Black, W. C. (2005). Análise multivariada de dados (5a ed.). Porto Alegre: Bookman.

Hanvanich, S., Sivakumar, K., \& Hult, T. M. (2006). The relationship of learning and memory with organizational performance: the moderating role of turbulence. Journal of the Academy of Marketing Science, 34(4), 600-612.

Hurley, R. F., \& Hult, T. M. (1998). Innovation, market orientation, and organizational learning: an integration and empirical examination. Journal of Marketing, 62(3), 42-54.

Kohli, A. K., \& Jaworski, B. (1990). Market orientation: the construct, research propositions, and managerial implications. Journal of Marketing, 54(2), 1-18.

Kohli, A. K., \& Jaworski, B. (1993). Market orientation: antecedents and consequences. Journal of Marketing, 57(3), 53-70.

Kohli, A. K., Jaworski, B., \& Kumar, A. (1993). Markor: a measure of market orientation. Journal of Marketing Research, 30(4), 467-477.

Malhotra, N. K. (2004). Pesquisa de marketing: uma orientação aplicada (4a. ed.). Porto Alegre: Bookman.

Narver, J. C., \& Slater, S. F. (1990). The effect of market orientation on business profitability. Journal of Marketing, 54(4), 20-35.

Perin, M. G. (2001). A relação entre orientação para o mercado, aprendizagem organizacional $e$ performance (Tese de doutorado). Universidade Federal de Rio Grande do Sul, Porto Alegre, RS, Brasil.

Perin, M. G., Sampaio, C. H., \& Faleiro, S. N. (2004). O impacto da orientação para o mercado e da orientação para aprendizagem sobre a inovação de produto: uma comparação entra a indústria eletroeletrônica e o setor de ensino universitário de administração. Revista de Administração Contemporânea, 8(1), 79-103.

Revista de Administração e Inovação, São Paulo, v. 10, n.3, p.65-88, jul./set. 2013. 
Perin, M. G, Sampaio, C. H., Baker, W. E., \& Simões, C. (2009, setembro). Capital social, empreendedorismo, geração de informação do mercado e a inovação radical. Anais do Encontro da Anpad, São Paulo, SP, Brasil, 33.

Ringle, C. M., Wende, S., \& Will, A. (2005). SmartPLS 2.0 M3 (beta). Germany: University of Hamburg. Recuperado de: http://www.smartpls.de.

Sinkula, J. M., Baker, W. E., \& Noordewier, T. (1997). A framework for market-based organizational learning: linking values, knowledge, and behavior. Journal of the Academy of Marketing Science, 25(4), 305-318.

Sistema Integrado de Informações sobre Operações Interestaduais com Mercadorias e Serviços. (2010). Consulta Pública ao Cadastro do Estado do Rio Grande do Sul. Recuperado em 18 de setembro, 2010, de: http://www.sintegra.gov.br.

\title{
STRATEGIC ORIENTATIONS AND PERFORMANCE: EVIDENCES FROM RETAIL OPTICAL COMPANIES
}

\begin{abstract}
The market and learning orientations interfere on organizational performance by direct impacts on innovation (Baker \& Sinkula, 1999). The aim of this study was to evaluate the market and learning orientation, innovation and the impact of these variables on the performance of optical organizations of Passo Fundo/RS. This study characterizes as a survey with the accession of 24 optics. The data were analyzed by using descriptive and multivariate analysis. In multivariate analysis and structural equation modeling, the estimation method used was Partial Least Squares (PLS). The results suggest that the market orientation and the innovation impact positively on performance. One hand, it was observed that the learning orientation impacts positively the market orientation. On the other hand, learning orientation has a negative impact on innovation. It is considered that additional studies are needed in specific business sectors.
\end{abstract}

Keywords: Organizational performance; innovation; learning orientation; market orientation.

Data do recebimento do artigo: 06/11/2012

Data do aceite de publicação: 15/06/2013

Revista de Administração e Inovação, São Paulo, v. 10, n.3, p.65-88, jul./set. 2013. 